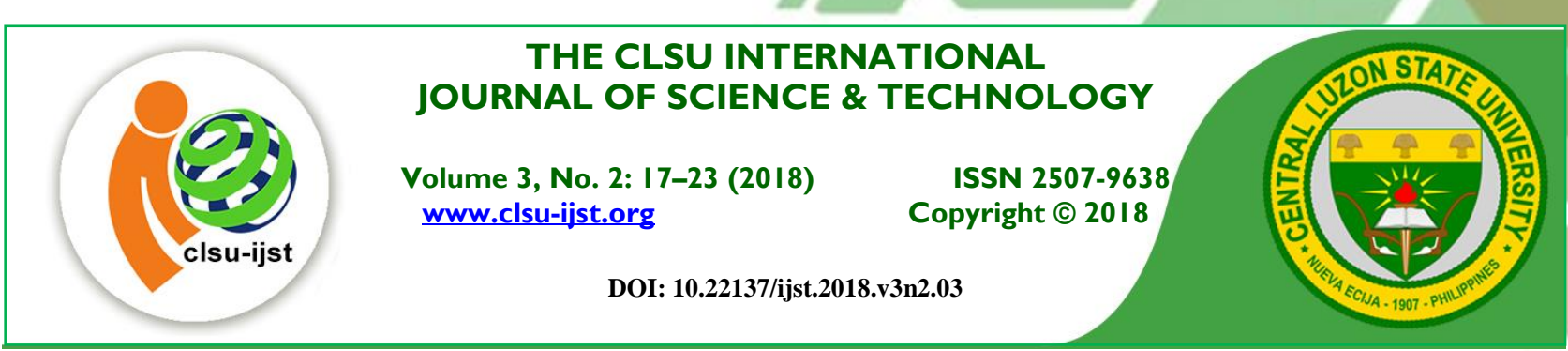

\title{
Myco-Chemicals and Teratogenic Activity of Wild Mushroom Trichaleurina celebica from Mt. Palali, Quezon, Nueva Vizcaya, Luzon Island, Philippines
}

\author{
Maricris M. Sogan ${ }^{1}$, Jason Arnold L. Maslang ${ }^{1}$, Rich Milton R. Dulay ${ }^{2 *}$
}

\begin{abstract}
${ }^{1}$ Center for Natural Sciences, School of Arts and Sciences, Saint Mary's University, Bayombong, Nueva Vizcaya, Philippines

${ }^{2}$ Bioassay Laboratory, Department of Biological Sciences, College of Arts and Science, Central Luzon State University, Science City of Muñoz, Nueva Ecija, Philippines

*E-mail for correspondence: richmiltondulay@clsu.edu.ph
\end{abstract}

\begin{abstract}
Trichaluerina celebica or lateg-lateg among Ilocanos of the Philippines is a wild edible mushroom that grows singly or in clusters on fallen logs. This study determined the embryo-toxic and teratogenic effects of extract of fruiting bodies of T. celebica on the developing zebrafish (Danio rerio) embryos and elucidated the secondary metabolites. Extract at $2 \%$ or higher concentrations of T. celebica caused $100 \%$ mortality rate of zebrafish embryo with coagulation as the most distinct toxic effect. Embryos exposed at $0.5 \%$ and $1 \%$ concentrations showed delayed hatching process resulting to the presence of abnormalities. The distinct teratogenic effects of T. celebica were tail malformations, delayed growth, and head deformation. The effects were found dependent on the concentration and time of exposure. Further, the fruiting bodies of T. celebica contain alkaloids, fatty acids, triterpenes, sterols, steroids, anthraquinones, anthrones, and steroids. Altogether, T. celebica has promising bioactivities which can be utilized in the nutraceutical or pharmacological industry.
\end{abstract}

Keywords: secondary metabolites, teratogenicity, Trichaleurina celebica, zebrafish embryo.

\section{INTRODUCTION}

Mushrooms as source of food are said to be rich in proteins, vitamins and minerals, and other beneficial constituents. They are also a valuable source of various bioactive substances like antibacterial, antioxidant, antiviral, anti-inflammatory, anti-cancer, anti-tumor, anti-HIV (Zhong and Xiao 2009; Oyetayo et al. 2012; De Silva et al. 2013), cytotoxic, anticoagulant, cholesterol-lowering, immunostimulatory, and hepato-protective compounds (Lindequist et al. 2005; Oyetayo et al. 2012). Although mushrooms are great source of therapeutic advantages, some constituents are known to be potentially toxic and teratogenic (Bustillos et al. 2016) especially those poisonous mushrooms belonging to the Amanita, Inocybe, Panaeolus, and Russulaceae species. The toxic components may be poisonous while teratogenic compounds may cause deformities to developing embryos. 


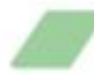

The Philippines is indisputably home to diverse natural resources which include mushrooms. Mt. Palali in Quezon, Nueva Vizcaya, for example, is one of the most explored places for its mushroom diversity. Among these promising macrofungi is Trichaleurina celebica, a saprobic mushroom that grows on decaying hardwood sticks and logs. It either grows singly or in clusters usually during wet seasons. This mushroom species which was formerly known as Galiella celebica was subjected to molecular identification by Valdez and Maslang (2015) and was found to be similar with T. celebica with $87 \%$ identity match on NCBI online BLAST. Furthermore, in the recent study of Maslang et al. (2015), this mushroom species was found to have numerous potential compounds. Subsequently, Matias et al. (2016) successfully reported the angiogenic and anti-tumor potential of this mushroom.

With the reported functional properties of T. celebica, it is therefore considered a promising alternative for various diseases. To date, there are still no studies regarding the embryo-toxicity and teratogenicity of $T$. celebica, thus, this study. The secondary metabolites present in this mushroom were also elucidated.

\section{MATERIALS AND METHODS}

Collection of mushroom. Fruiting bodies of T. celebica were collected from Mt. Palali, Quezon, Nueva Vizcaya. The collected mushroom species were washed, sliced, and air-dried for at most 5 days prior to extraction for the assay and analysis of the myco-chemical constituents.

Extraction of functional components. Samples were pulverized using a blender and 20 grams of the powdered mushroom was dissolved in $600 \mathrm{ml}$ distilled water and maintained at $80-90 \mathrm{oC}$ for 2 hours in water bath. The extract was filtered using Whatman filter paper No. 2. Extract filtrate was diluted to embryo water to prepare the different concentrations for the embryo-toxicity and teratogenicity assay.

Spawning of zebrafish. The procedure in spawning zebrafish was adopted from the study of Dulay et al. (2014). Adult zebrafish (6 males and 8 females) were acclimatized in an aquarium filled with non-chlorinated water and were confined in a plastic mesh in order to prevent the adult fish from devouring their eggs. Subsequently, the process of spawning was done by incubating the fish first in dark conditions for 12 hours. After the first phase of incubation which induced spawning, the aquarium was exposed to light in order for fertilization to be stimulated (Dulay et al. 2012; Dulay et al. 2014). The fertilized eggs were collected and washed in a petri plate prior to examination under microscope to determine that the eggs were normal and at segmentation phase before subjecting them to experimental treatment.

Embryo-toxicity and teratogenicity test. The methods used for the embryo-toxicity and teratogenicity assay followed the procedures of Dulay et al. (2014). Two $\mathrm{ml}$ of each treatment concentration of extract $(3 \%, 2 \%, 1 \%, 0.5 \%$, and $0.1 \%$ ) and control (embryo water) were dispensed into each well of the 12-well ELISA plate. Four embryos at 12-hour post fertilization (hpf) were transferred into each well containing the extract concentrations and incubated at room temperature. Three replicates were done in this study. The embryos were then examined at time points 12, 24, 36, and 48 hour post treatment application (hpta) for the mortality, hatchability, and the presence of morphological abnormalities.

Detection of secondary metabolites through thin layer chromatography. The secondary metabolites present in the hot water extract of T. celebica were detected through TLC plates. The different spray reagents were used in order to identify the different secondary metabolites of mushroom. First, measurements were done on TLC plates to serve as guidelines of sample spot and Vol. 3 No. 2 ISSN: 2507-9638 DOI: 10.22137/ijst.2018.v3n2.03

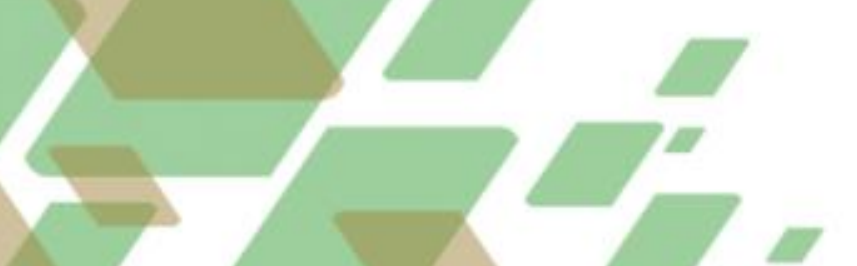


solvent front. A capillary tube with straightcut edges was dipped into the extract container and immediately spotted on the TLC-coated plates and were air-dried afterwards. Then, the plates were placed inside an equilibrated developing chamber with ethyl acetate as the developing solvent and covered to allow the solvent to travel upwards until it reached the mark at the top of the TLC plates. The developing chromatograms were removed from the chamber and dried using the hot plate. To detect the presence of the desired constituents, each plate was dipped into the suitable reagents and were viewed with/without UV light. The results were evaluated by phytochemical screening procedures where positive results were indicated by the different colorations in the form of spots which manifested on the TLC plates.

Statistical analysis. Data were analyzed using Analysis of Variance (ANOVA) and compared using Duncan Multiple Range Test (DMRT) at 5\% level of significance.

\section{RESULTS AND DISCUSSION}

Toxic effects of $T$. celebica extract. Using the zebrafish embryos, the toxic effects of $T$. celebica extract were evaluated. The percentage mortality of embryos after exposure to the various treatments at observation time points is shown in Table 1. It was found out that the toxicity of the extract is dependent on the dose and time of exposure to the extract. At 12 hpta, zero mortality was observed from all the extracts. After further exposure for 24 hours, mortality rate of $16.67 \%$ for both $2 \%$ and $3 \%$ was observed which significantly increased after 36 hours of exposure with recorded mortality rate of $75 \%$ and $41.67 \%$ for $3 \%$ and $2 \%$ concentration, respectively. The toxic effect of the mushroom extract was fully manifested after 48 hours in $2 \%$ and 3\% concentration, which recorded the highest mortality of $100 \%$ as evident by the coagulation of the embryos. Moreover, it can be observed that the embryos at these two concentrations clearly displayed no visible structures. Mortality was not observed in embryos exposed to lower concentrations at similar time point. However, signs of morphological deformities such as stunted growth were noticeable at $1 \%$ extract concentration. The presence of these malformations may have been the contributing factor which caused the mortality of hatched embryos exposed at $0.5 \%$ and $1 \%$ after further exposure to the extract. On the other hand, untreated embryos and embryos exposed to $0.1 \%$ concentration showed normal development. Apparently, extract of mushroom is lethal at $2 \%$ concentration and higher after prolonged exposure resulting to more coagulated embryos. The results of the present study regarding the toxic effect of $T$. celebica may be due to the different bioactive compounds present in its aqueous extract. Recently, Matias et al. (2016) and Maslang et al. (2015) both reported that extract of T. celebica had high cytotoxic property using brine shrimp as model and it is possible that the present bioactive compounds in this macrofungus contributed to its reported cytotoxicity. 

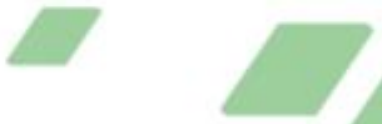

Table 1. Mortality of zebrafish embryos after 12, 24, 36, and 48 hours of exposure at different concentrations of T. celebica extract.

\begin{tabular}{lccccc}
\hline \multirow{2}{*}{ Treatment } & Concentration & \multicolumn{4}{c}{ Mortality $(\%)$} \\
\cline { 3 - 6 } & $(\%)$ & 12 hours & 24 hours & 36 hours & 48 hours \\
\hline Control & 0.0 & 0.00 & $0.00^{\mathrm{b}}$ & $0.00^{\mathrm{c}}$ & $0.00^{\mathrm{b}}$ \\
T. celebica Extract & 0.1 & 0.00 & $0.00^{\mathrm{b}}$ & $0.00^{\mathrm{c}}$ & $0.00^{\mathrm{b}}$ \\
& 0.5 & 0.00 & $0.00^{\mathrm{b}}$ & $0.00^{\mathrm{c}}$ & $0.00^{\mathrm{b}}$ \\
& 1.0 & 0.00 & $0.00^{\mathrm{b}}$ & $0.00^{\mathrm{c}}$ & $0.00^{\mathrm{b}}$ \\
& 2.0 & 0.00 & $16.67^{\mathrm{a}}$ & $41.67^{\mathrm{b}}$ & $100.00^{\mathrm{a}}$ \\
& 3.0 & 0.00 & $16.67^{\mathrm{a}}$ & $75.00^{\mathrm{a}}$ & $100.00^{\mathrm{a}}$ \\
\hline
\end{tabular}

Values are treatment means. Means with the same letter of superscript are not significantly different from each other at $5 \%$ level of significance using DMRT.

Teratogenic effects of T. celebica extract. The teratogenic effects of the extract was evaluated based on the morphological abnormalities including tail malformation, head deformation and delayed development. The different morphological abnormalities are shown in Figure 1. It can be noticed that embryos under the normal condition and those exposed to $0.1 \%$ concentration of the extract showed normal development as compared to those embryos treated at higher concentrations. Since embryos treated with $2 \%$ and $3 \%$ concentration manifested $100 \%$ coagulation, only those embryos treated with 0.5 and $1 \%$ of the extract were examined for presence of malformations. In this study, the most marked teratogenic endpoint of $T$. celebica extract is tail malformation as shown by the presence of kinks or the bending of tails on concentrations $0.5 \%$ and $1 \%$. Aside from tail abnormalities, some embryos incubated at $0.5 \%$ and $1 \%$ extract concentration showed presence of head abnormalities. Moreover, growth retardation which might have contributed to the delay of the hatching process of embryos in $0.5 \%$ and $1 \%$ concentrations is also considered a teratogenic effect of the extract. Similar manifestations of teratogenic effects on zebrafish embryos were found as effects of other mushrooms such as Panaeolus, Lentinus tigrinus, Ganoderma lucidum, Letinus sajor-caju, and Pleurotus ostreatus (Dulay et al. 2012; Dulay 2014; De Castro and Dulay, 2015; Bustillos et al. 2016). In addition, in the study of Adewusi et al. (1993), Termitomyces robustus and Volvariella esculenta inhibited the growth and showed $100 \%$ mortality using rat models. Apparently, the teratogenic effect of T. celebica extract is dosedependent. Similarly with the lethal effect, the bioactive components of T. celebica may as well have played a role for the teratogenic effects of the extracts. The secondary metabolites present in the extract could have caused the morphological abnormalities observed in the zebrafish larvae. Studies have reported that some secondary metabolites from plants are fetotoxic which can cause abortion and birth defects in offspring (Forbey et al. 2009).

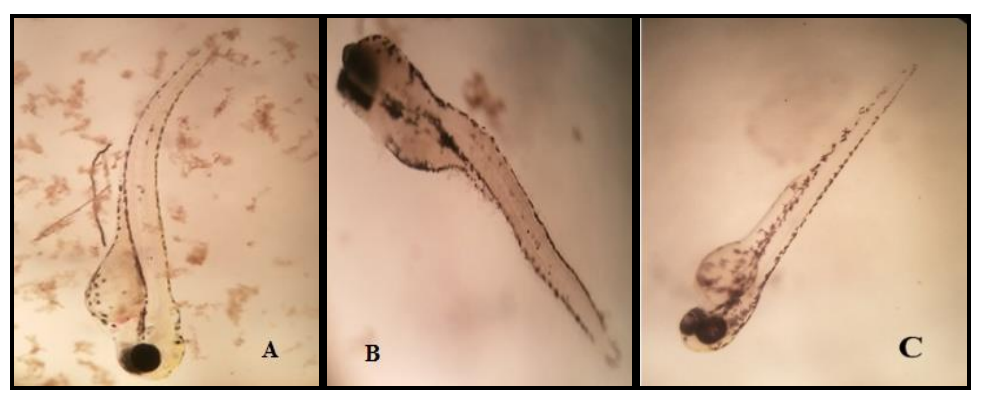

Figure 1. Morphological abnormalities of the zebrafish showing tail malformation and deformation of head of larvae after 72 hpta. Bent-tail hatched larva exposed at $0.5 \%$ (A) Kinks in the tails, sidewise position and head deformation detected at $1 \%$; (B) concentration of the mushroom extract; (C) Normal embryo.

Vol. 3 No. 2 ISSN: 2507-9638 DOI: 10.22137/ijst.2018.v3n2.03 


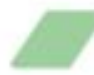

Hatchability of $D$. rerio. One of the indications of successful embryonic developmental process is the hatchability of the embryos that is influenced by their ability to rupture the protective chorion (Romagosa et al. 2016). The percentage hatchability of embryos after 48 hours of extract exposure is presented in Table 2. As compared to embryos treated with higher concentrations of the mushroom extract, all control embryos and those at $0.1 \%$ of extract were able to hatch and develop normally. The delay in the hatching of embryos treated with $0.5 \%$ and $1 \%$ concentration which took place after three days of exposure may have been affected by the observed malformations among the embryos. This is similar with the effect of Ganoderma lucidum and Termitomyces clypeatus (Dulay et al. 2012; De Castro et al. 2016) such that morphological abnormalities limited the ability of embryos to break off the chorion prior to hatching. For $2 \%$ and $3 \%$, however, hatchability was no longer evaluated because of embryo mortality. As previously noted by other similar studies, the hatchability of zebrafish embryos is greatly affected by the concentration of the extract which decreases as the extract concentration increases.

Table 2. Hatchability of zebrafish after 48 hours of exposure to the different extract concentrations of T. celebica.

\begin{tabular}{lcc}
\hline Treatment & Concentration $(\%)$ & Hatchability $(\%)$ \\
\hline Control & 0.0 & $100.00^{\mathrm{a}}$ \\
T. celebica Extract & 0.1 & $100.00^{\mathrm{a}}$ \\
& 0.5 & $58.33^{\mathrm{b}}$ \\
& 1.0 & $0.00^{\mathrm{c}}$ \\
& 2.0 & $0.00^{\mathrm{c}}$ \\
& 3.0 & $0.00^{\mathrm{c}}$ \\
\hline
\end{tabular}

Values are treatment means. Means with the same letter of superscript are not significantly different from each other at $5 \%$ level of significance using DMRT.

Chemical constituents of $T$. celebica. In this study, the secondary metabolites that may have contributed to the embryo-toxicity and teratogenicity of T. celebica were determined from its aqueous extract and are presented in Table 3. In the previous study of Maslang et al. (2015), secondary metabolites such as coumarins, anthrones, alkaloids, phenolics, flavonoids, steroids, and anthraquinones were detected from the methanolic crude extract of the mushroom fruiting body. The phytochemical screening revealed that certain metabolites like alkaloids (showed an intense orangebrown coloration), anthraquinones, anthrones, fatty acids, higher alcohols, phenols, steroids, and triterpenes are present in the aqueous extract. Since the extracting solvent used was water, it is expected that only polar compounds will be extracted which includes alkaloids and glycosides (Hanson, n.d.). However, since water is a universal solvent, other compounds such as water-soluble flavonoids and phenolic were traced but their efficacy when used in antimicrobial examination usually degrade as compared to extracts from organic solvents (Tiwari et al. 2011). Though the role of the extracted secondary metabolites in the embryo-toxicity and teratogenicity of the extract of $T$. celebica is not readily conclusive, these compounds, especially those known to be toxic, alkaloids, for example, may have combined effects with one another. Thus, the isolation of the specific compounds that causes the teratogenicity and embryo-toxicity should be done in future studies. 
Table 3. Chemical compositions of T. celebica.

\begin{tabular}{lll}
\hline Spray Reagents & Positive Indicator & Detected Chemical \\
\hline Preliminary Test & Violet spot under UV 365 nm & Essential oils \\
Vanillin sulphuric acid & Yellow spots & Fatty acids \\
& Blue-violet spots under UV 365 nm & Triterpenes and sterols \\
& Brown spot & Phenols \\
Methanolic KOH & Orange coloration at visible light & Anthraquinones \\
& Yellow zones under UV 365 nm & Anthrones \\
Dragendorff reagent & Brown-orange spots & Alkaloids \\
Antimony (III) chloride & Fluorescent colors under UV 365 nm & Steroids \\
\hline
\end{tabular}

In conclusion, hot water extract of $T$. celebica is toxic and teratogenic to zebrafish embryos as shown by high mortality rate, tail malformations, head deformation, delayed growth, and low percentage of hatchability. The findings indicate that as an edible mushroom, T. celebica must be eaten with precaution, and, as a potential therapeutic mushroom, it can also be a significant source of toxic and teratogenic compounds which could be further studied for the development of pharmaceutical products like anti-cancer drugs.

\section{REFERENCES}

Adewusi, S., F. Alofe, O. Odeyemi, O. Afolabi, and O. Oke. 1993. "Studies on Some Edible Wild Mushrooms from Nigeria: Nutritional, Teratogenic, and Toxic Consideration." Plant Foods for Human Nutrition 43(2): 115-121.

Bustillos, R., Z. Paguio, D. Hermosa, and R.M. R. Dulay. 2016. "Philippine Coprophilous Mushrooms (Panaeolus antillarium and Panaeolus cyanescens) Exhibit Toxic and Teratogenic Effects in Zebrafish (Danio rerio) Embryo Model." Advances in Environmental Biology 10(3): 75-80.

De Castro, M. E. G., R. M. R. Dulay, and M. L. Enriquez. 2016. "Toxic and Teratogenic Effects of Medicinal and Culinary Mushroom, Termitomyces clypeatus, Collected from the Termite Mound in Mt. Makiling Forest Reserve, Los Baños, Laguna, Philippines on Developing Embryos Of Zebrafish (Danio rerio)." Der Pharmacia Lettre 8(5): 237-242.

De Castro, M. E. G., and R. M. R. Dulay. 2015. "Toxic and Teratogenic Effects of Lentinus sajor-caju and Pleurotus ostreatus Ethanolic Extracts in Danio rerio Embryo Model." International Journal of Biology, Pharmacy, and Allied Sciences 4(4): 2261-2269.

De Silva, D., S. Rapior, E. Sudarman, M. Stadler, J. Xu, A. Alias, and K. Hyde. 2013. "Bioactive Metabolites from Macrofungi: Ethnopharmacology, Biological Activities, and Chemistry." Fungal Diversity 62: 1-40.

Dulay, R. M. R., S. P. Kalaw, R. G. Reyes, N. Alfonso, and F. Eguchi. 2012. "Teratogenic and Toxic Effects of Lingzhi or Reishi Medicinal Mushroom, Ganoderma lucidum, (W. Curt.:Fr.) P. Karts. (Higher Basidiomycetes), on Zebrafish Embryo as Model." International Journal of Medicinal Mushrooms 14(5): 507512. 


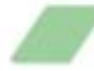

Dulay, R. M. R., S. P. Kalaw, R. G. Reyes, and E. Cabrera. 2014. "Embryo-Toxic and Teratogenic Effects of Philippine Strain of Lentinus tigrinus (Tiger Sawgill Basidiomycetes) Extract on Zebrafish (Danio rerio) Embryos." Annals of Biological Research 5(6): 9-14.

Forbey, J., A. Harvey, M. Huffman, F. Provenza, R. Sullivan, and D. Tasdemir. 2009. "Exploitation of Secondary Metabolites by Animals: A Response to Homeostatic Challenges." Integrated Comparative Biology 49(3): 314-328.

Hanson, J. R. n.d. Natural Products: The Secondary Metabolites. http://pubs.rsc.org/en/Content/eBook/978-0-85404-490-0\#!divbookcontent

Lindequist, U., T. Niedermeyer, and W. Julich. 2005. "Pharmacological Potentials of Mushroom." Evidence-Based Complementary and Alternative Medicine 2(3): 285-299.

Maslang, J., C. Matias, M. Bungihan, M. Saludarez, D. Liday, H. Villanueva, M. Balonquita, F. Cabading, and S. Valdez. 2015. "Mycelial Propagation of Trichaluerina celebica with Analysis of its Secondary Metabolites, Nutritional Composition, Antibacterial, Anti-inflammatory, Antioxidant, and Cytotoxic properties.” Undergraduate Thesis. Saint Mary's University.

Matias, C., J. Maslang, M. Bungihan, M. Saludarez, D. Liday, H. Villanueva, M. Balonquita, and M. Mariñas. 2016. "Isolation of the Cytotoxic, Angiogenic, and Anti-tumor compounds from Trichaluerina celebica. Undergraduate Thesis. Saint Mary’s University.

Oyetayo, O. V., A. N. Camacho, T. M. Apana, R. E. Baldomero, and M. Jimenez. 2012. Total Phenol, Antioxidant, and Cytotoxic Properties of Wild Macrofungi Collected from Akure Southwest Nigeria." Jordan Journal on Biological Science 6(2): 105-118.

Romagosa, C., E. David, and R. M. R. Dulay. 2016. "Embryo-toxic and Teratogenic Effects of Tinospora cordifolia Leaves and Bark Extracts in Zebrafish (Danio rerio) Embryos." Asian Journal on Plant Sciences and Research 6(2): 37-41.

Tiwari, P., B. Kumar, M. Kaur, G. Kaur, and H. Kaur. 2011. "Plant Screening and Extraction: A Review." Internationale Pharmacentica Sciencia 1(1): 98-106.

Valdez, S. J., and J. Maslang. 2015. "Morphological Characteristics, Molecular Profile, and an In-vitro Anti-inflammatory Activity of Trichaleurina celebica." Undergraduate Thesis. Saint Mary's University.

Zhong, J., and J. Xiao. 2009. "Secondary Metabolites from Higher Fungi: Discovery, Bioactivity, and Bioproduction." Advances in Biochemical Engineering 113: 79-150. 